\title{
On a method of image reconstruction of anisotropic media using applied quasipotential tomographic data
}

\author{
Bomba A. Ya., Kuzlo M. T., Michuta O. R., Boichura M. V. \\ National University of Water and Environmental Engineering, \\ 11 Soborna Str., 33028, Rivne, Ukraine
}

(Received 18 July 2019; Revised 28 October 2019; Accepted 5 November 2019)

\begin{abstract}
An algorithm for solving the coefficient problems of parameter identification of anisotropic media using applied quasipotential tomographic data is modified for the case of presence of more specific a priori information about the eigendirections of the corresponding conductivity tensor. Its application is quite common in practice, in particular, in medicine, where the object of such study may be the medium with fibrous or layered areas (which includes muscles, bones, etc.), inside which there are streams of non-spherical particles (e.g. red blood cells). As in our previous works, the corresponding algorithm is based on alternately solving the quasiconformal mapping and parameter identification problems, but in this work it is supplemented by the procedure of parallelization of calculations and the optimization problem is "accelerated". The latter is characterized by a significant decrease in the number of intermediate calculations and, when imposing additional restrictions on eigendirections of the conductivity tensor, leads to the possibility of optimal adaptation of the algorithm to specific cases of practice. The results of numerical experiments of imitative restoration of medium structure are presented.
\end{abstract}

Keywords: applied quasipotential tomography, quasiconformal mappings, anisotropy, identification, nonlinear inverse problems.

2000 MSC: 30C62, 35J25, 65E05, 65N21

UDC: 519.6

DOI: $10.23939 / \mathrm{mmc} 2019.02 .211$

\section{Introduction}

Electrical impedance tomography is a relatively new method of visualizing the interior of the investigated object, which is characterized by low cost, non-invasiveness, geometric flexibility, environmental friendliness, etc. [1]. Due to this, it has widely proliferated into a large number of branches of science and technology [1-5]. The main drawback of the method is the low resolution of the obtained images. This is a consequence of significant mathematical simplifications in comparison to the statement of Calderon's problem [6]. In particular, in [1-5,7] etc. the input and output sections of the investigated body are considered to be point-like (although with finite values of the potential). We have published a series of works in which the methodology for image reconstruction of the investigated object interior using applied quasipotential tomographic (AQT) data (see, e.g. $[8,9]$ ), provided that the distribution of local velocity values along the boundary input and output sections is taken into account, is proposed. In particular, in [9] the numerical quasiconformal mapping method is generalized for solving the coefficient problems of finding the eigenvalues of the conductivity tensor (CT) in cases where information about its directions in an anisotropic medium is available. However, the synthesis of the numerical quasiconformal mapping method, ideas of alternating block parametrization and the corresponding difference analogues of the AQT problem are not satisfactorily covered there. The synthesis problem, which lies in minimizing the functional of the sum of the squares of the residuals of the expressions obtained from the generalized Cauchy-Riemann conditions, using the regularization concept and provided that the eigenvalues are positive, can be improved both in reducing the number of calculations of the objective function and limiting the set of permissible solutions. The latter is useful in cases when information about the directions of larger ellipse axis is available (instead of any of them, as was in [9]). 
In other words, the aim of this paper is to generalize the numerical quasiconformal mapping method [10] for solving the coefficient problems of finding the eigenvalues of the CT having additional information about their eigendirections in an anisotropic medium using AQT data [9], and for reduce the number of calculations by the way of parallelizing the computations and refining the optimization procedure. This is the kind of statements that is typical in medical, geological imaging, etc.

\section{Identification of anisotropic media parameters using AQT data with application of complex analysis methods (problem statement)}

It is quite often possible to isolate a quasi-two-dimensional domain of the most intensive motion of particles (in particular, liquids, electric charges) when researching processes in electrical impedance tomography $[1,6,7,11]$. Usually the corresponding identification problem is solved in a single-connected curvilinear domain (anisotropic layer or plate, which is some tomographic cross-section) $G_{z}$ Fig. 1a, bounded by the smooth closed curve $\partial G_{z}=\{(x, y): x=\tilde{x}(\tau), y=\tilde{y}(\tau), 0 \leqslant \tau \leqslant 2 \pi, \tilde{x}(0)=\tilde{x}(2 \pi)=$

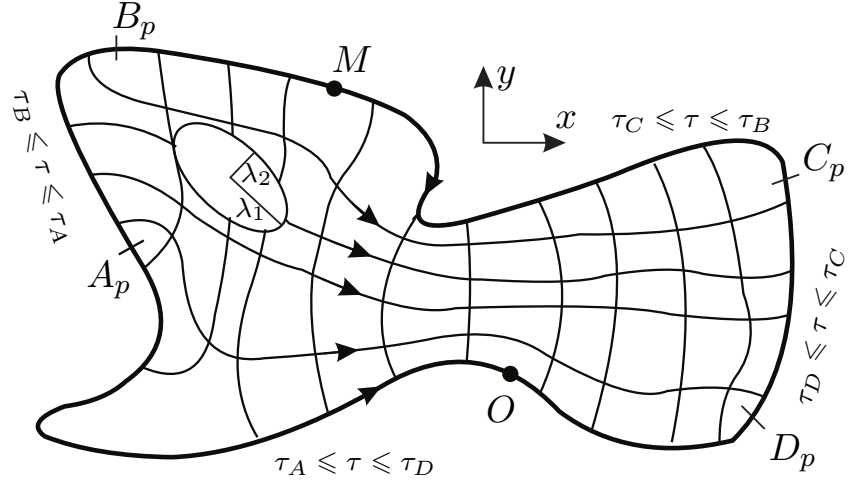

$\boldsymbol{a}$

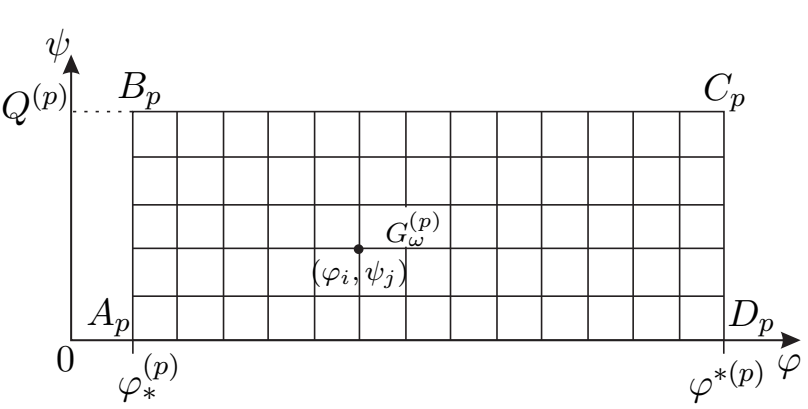

$b$

Fig. 1. Tomographic cross-section $G_{z}(\boldsymbol{a})$ and corresponding to the $p$ th injection complex quasipotential domain $G_{\omega}^{(p)}(\boldsymbol{b})$.

$\left.\tilde{x}_{0}, \tilde{y}(0)=\tilde{y}(2 \pi)=\tilde{y}_{0}\right\}$, where $\tilde{x}(\tau), \tilde{y}(\tau)$ are defined continuously differentiated functions, $O\left(\tilde{x}_{0}, \tilde{y}_{0}\right)$ is given reference point. It is also assumed that quasiideal processes of particles movement are present in $G_{z}$ having various situational states (injections), which are modeled by the generalized Laplace equation

$$
\frac{\partial}{\partial x}\left(\sigma_{11} \frac{\partial \varphi^{(p)}}{\partial x}+\sigma_{12} \frac{\partial \varphi^{(p)}}{\partial y}\right)+\frac{\partial}{\partial y}\left(\sigma_{21} \frac{\partial \varphi^{(p)}}{\partial x}+\sigma_{22} \frac{\partial \varphi^{(p)}}{\partial y}\right)=0
$$

and (according to the statement of electrical impedance tomography problem proposed by A. Calderon; to ensure uniqueness of the solution) an infinite number of interconnected conditions of Dirichlet and Neumann for each given $p[6,11]$. Here (1) is a consequence of the generalized motion law of Ohm, Darcy etc. $\boldsymbol{j}^{(p)}=\sigma \operatorname{grad} \varphi^{(p)}$ and the continuity equation $\operatorname{div} \boldsymbol{j}^{(p)}=0[1,10] ; \boldsymbol{j}^{(p)}(x, y)$ are current densities; $\varphi^{(p)}=\varphi^{(p)}(x, y)$ are quasipotentials; $p=1,2, \ldots$ is the injection number (see, e.g. $[3,5]) ; \sigma_{\alpha \beta}=\sigma_{\alpha \beta}(x, y, \ldots)$ are bounded continuously differentiated in the domain $G_{z}$ functions that characterize the conductivity and anisotropy of the medium $[1,3,7,11,12]$. In practice, the contact surface between electrode and investigated body has a finite size and it is often convenient to consider the sections of constancy of the corresponding potentials $[8,9]$. We, unlike $[1-5,7]$, also propose to set the local velocities distribution there and to take into account both the values of stream functions and the distribution of potentials at other sections $[8,9]$. Generally, it is also impossible to set an infinite number of these conditions. In practice, therefore, certain simplifications are allowed (see, e.g. [1,3,7]).

First of all, a finite number of current injections through the tomographic cross-section is considered. They, similar to $[8,9]$, will be modeled by sets of values $\left\{\tau_{A}^{(p)}, \tau_{B}^{(p)}, \tau_{C}^{(p)}, \tau_{D}^{(p)}\right\}$, which is equivalent 
to setting four points $A_{p}=\left(\tilde{x}\left(\tau_{A}^{(p)}\right), \tilde{y}\left(\tau_{A}^{(p)}\right)\right), B_{p}=\left(\tilde{x}\left(\tau_{B}^{(p)}\right), \tilde{y}\left(\tau_{B}^{(p)}\right)\right), C_{p}=\left(\tilde{x}\left(\tau_{C}^{(p)}\right), \tilde{y}\left(\tau_{C}^{(p)}\right)\right), D_{p}=$ $\left(\tilde{x}\left(\tau_{D}^{(p)}\right), \tilde{y}\left(\tau_{D}^{(p)}\right)\right)$. According to given injection, the boundary of the domain $G_{z}$ with given four marked points is denoted by $\partial G_{z}^{(p)}\left(z^{(p)}=x^{(p)}+i y^{(p)}\right)$. In this case, we rewrite a mathematical model of AQT [1], similar to $[8,9]$, in the form (1) and with the following conditions:

$$
\begin{aligned}
& \left.\varphi^{(p)}\right|_{A_{p} B_{p}}=\varphi_{*}^{(p)},\left.\quad \varphi^{(p)}\right|_{C_{p} D_{p}}=\varphi^{*(p)},\left.\quad j^{(p)}\right|_{B_{p} C_{p} \cup A_{p} D_{p}}=0,\left.\quad \varphi^{(p)}(M)\right|_{A_{p} D_{p}}=\underline{\varphi}^{(p)}(M), \\
& \left.\varphi^{(p)}(M)\right|_{B_{p} C_{p}}=\bar{\varphi}^{(p)}(M),\left.\quad j^{(p)}(M)\right|_{A_{p} B_{p}}=\Psi_{*}^{(p)}(M),\left.\quad j^{(p)}(M)\right|_{C_{p} D_{p}}=\Psi^{*(p)}(M),
\end{aligned}
$$

where $A_{p} B_{p}$ and $C_{p} D_{p}$ are selected equipotential lines; $B_{p} C_{p}$ and $A_{p} D_{p}$ are impermeable boundary streamlines; $\boldsymbol{n}$ is a unit outward normal vector; $M$ is a running point on the corresponding curve. Functions $\underline{\varphi}^{(p)}(M)=\underline{\varphi}^{(p)}(\tau, \ldots)\left(\tau_{A}^{(p)} \leqslant \tau \leqslant \tau_{D}^{(p)}\right), \bar{\varphi}^{(p)}(M)=\bar{\varphi}^{(p)}(\tau, \ldots)\left(\tau_{C}^{(p)} \leqslant \tau \leqslant \tau_{B}^{(p)}\right), \Psi_{*}^{(p)}(M)=$ $\Psi_{*}^{(p)}(\tau, \ldots)\left(\tau_{B}^{(p)} \leqslant \tau \leqslant \tau_{A}^{(p)}\right), \Psi^{*(p)}(M)=\Psi^{*(p)}(\tau, \ldots)\left(\tau_{D}^{(p)} \leqslant \tau \leqslant \tau_{C}^{(p)}\right)$, as in [8,9], can be constructed by interpolating the experimental obtained their values $\bar{\varphi}_{\bar{i}^{(p)}}^{(p)}, \underline{\varphi}_{\underline{\varphi}^{(p)}}^{(p)}, \Psi_{* j_{*}^{(p)}}^{(p)}, \Psi_{j^{*(p)}}^{*(p)}$ having some given $\tau=\bar{\tau}_{\bar{i}^{(p)}}^{(p)}, \tau=\underline{\tau}_{\underline{i}^{(p)}}^{(p)}, \tau=\tau_{* j_{*}^{(p)}}^{(p)}, \tau=\tau_{j^{*(p)}}^{*(p)}$ at the sections $B_{p} C_{p}, A_{p} D_{p}, A_{p} B_{p}, C_{p} D_{p}$, respectively $\left(\Psi_{* j_{*}^{(p)}}^{(p)}, \Psi_{j^{*(p)}}^{*(p)}>0, \varphi_{*}^{(p)} \leqslant \underline{\varphi}_{\underline{i}^{(p)}}^{(p)} \leqslant \varphi^{*(p)}, \varphi_{*}^{(p)} \leqslant \bar{\varphi}_{\bar{i}^{(p)}}^{(p)} \leqslant \varphi^{*(p)}, 0 \leqslant \bar{i}^{(p)} \leqslant \bar{m}^{*(p)}+1,0 \leqslant \underline{i}^{(p)} \leqslant \underline{m}_{*}^{(p)}+1\right.$, $\left.0 \leqslant j_{*}^{(p)} \leqslant n_{*}^{(p)}+1,0 \leqslant j^{*(p)} \leqslant n^{*(p)}+1\right)$. CT components with equal additional diagonal elements (see, e.g. $[3,4,9,11])$ are defined as follows:

$$
\sigma_{11}=\left(\lambda_{1}-\lambda_{2}\right) \cos ^{2} \theta+\lambda_{2}, \quad \sigma_{22}=\left(\lambda_{2}-\lambda_{1}\right) \cos ^{2} \theta+\lambda_{1}, \quad \sigma_{12}=\left(\lambda_{1}-\lambda_{2}\right) \sin \theta \cos \theta=\sigma_{21},
$$

the eigenvalues $\lambda_{1}, \lambda_{2}$ corresponding to (1) matrix are sought in the form of

$$
\begin{aligned}
& \lambda_{1}=\lambda_{1}\left(x, y, a_{s_{a}, 0}, \ldots, a_{0, s_{a}}\right)=\sum_{k_{a}, r_{a}=0}^{s_{a}, k_{a}} \frac{x^{k_{a}-r_{a}} y^{r_{a}}}{a_{k_{a}-r_{a}, r_{a}}^{-1}} \\
& \lambda_{2}=\lambda_{2}\left(x, y, b_{s_{b}, 0}, \ldots, b_{0, s_{b}}\right)=\sum_{k_{b}, r_{b}=0}^{s_{b}, k_{b}} \frac{x^{k_{b}-r_{b}} y^{r_{b}}}{b_{k_{b}-r_{b}, r_{b}}^{-1}}
\end{aligned}
$$

and function $\theta=\theta(x, y)$, characterizing the direction of anisotropy is considered, similar to $[1,3,9]$, to be known a priori (here, in contrast to [9], its values characterize the directions of larger axes of corresponding ellipses, which is typical for medical, geological and other practical problems of nowadays). Here $a_{k_{a}-r_{a}, r_{a}}, b_{k_{b}-r_{b}, r_{b}}\left(k_{a}=0, \ldots, s_{a}, r_{a}=0, \ldots, k_{a}, k_{b}=0, \ldots, s_{b}, r_{b}=0, \ldots, k_{b}\right)$ are parameters defined during the process of problem solving.

The problem lies in image reconstruction of the CT. In addition, it is accompanied by calculations of the corresponding dynamic meshes and velocity fields.

Through the introduction of stream functions $\psi^{(p)}=\psi^{(p)}(x, y)$, which are complexly conjugated with $\varphi^{(p)}=\varphi^{(p)}(x, y)(p=\overline{1, \tilde{p}})$, similarly to [8-10], we can reduce (1)-(4) to the series of more general boundary value problems of quasiconformal mapping $\omega=\omega^{(p)}(z)=\varphi^{(p)}(x, y)+i \psi^{(p)}(x, y)$ of the physical domains $G_{z}^{(p)}$ Fig. $1 a$ to the relevant domains of complex quasipotential $G_{\omega}^{(p)}$ Fig. $1 b$ under conditions (3) and (4):

$$
\begin{gathered}
\left\{\begin{array}{l}
\sigma_{11} \frac{\partial \varphi^{(p)}}{\partial x}+\sigma_{12} \frac{\partial \varphi^{(p)}}{\partial y}=\frac{\partial \psi^{(p)}}{\partial y}, \\
\sigma_{21} \frac{\partial \varphi^{(p)}}{\partial x}+\sigma_{22} \frac{\partial \varphi^{(p)}}{\partial y}=-\frac{\partial \psi^{(p)}}{\partial x} ;
\end{array}\right. \\
\left.\varphi^{(p)}\right|_{A_{p} B_{p}}=\varphi_{*}^{(p)},\left.\quad \varphi^{(p)}\right|_{C_{p} D_{p}}=\varphi^{*(p)},\left.\quad \varphi^{(p)}(M)\right|_{A_{p} D_{p}}=\underline{\varphi}^{(p)}(M),\left.\quad \varphi^{(p)}(M)\right|_{B_{p} C_{p}}=\bar{\varphi}^{(p)}(M), \\
\left.\psi^{(p)}\right|_{A_{p} D_{p}}=0,\left.\quad \psi^{(p)}\right|_{B_{p} C_{p}}=Q^{(p)},\left.\quad \psi^{(p)}(M)\right|_{A_{p} B_{p}}=\psi_{*}^{(p)}(M),\left.\quad \psi^{(p)}(M)\right|_{C_{p} D_{p}}=\psi^{*(p)}(M) ;
\end{gathered}
$$

Mathematical Modeling and Computing, Vol. 6, No. 2, pp. 211-219 (2019) 


$$
\int_{M N} j^{(p)} d l=Q^{(p)}, \quad M \in B_{p} C_{p}, \quad N \in A_{p} D_{p}
$$

where $G_{\omega}^{(p)}=\left\{(\varphi, \psi): \varphi_{*}^{(p)} \leqslant \varphi \leqslant \varphi^{*(p)}, 0 \leqslant \psi \leqslant Q^{(p)}\right\} ; \psi_{*}^{(p)}(M)=\int_{A_{p} M} \Psi_{*}^{(p)}\left(M_{l}\right) d l, \psi^{*(p)}(M)=$ $\int_{D_{p} M} \Psi^{*(p)}\left(M_{l}\right) d l ; Q^{(p)}$ are discharges of a vector field (current) through contact surfaces $\left(A_{p} B_{p}\right.$ and $\left.C_{p} D_{p}\right) ; d l$ and $M, M_{l}$ are an arc element and running points of the corresponding curves.

\section{Quasiconformal mapping inverse problem, its difference analogue and solving algorithm}

In view of the complexity of realization of mapping such as $G_{z}^{(p)} \rightarrow G_{\omega}^{(p)}$ (curvilinear physical domains to canonical domains of complex quasipotential), it is reasonable to consider the corresponding inverse ones [10]. Then the problem of AQT lies in the search of the functions $x^{(p)}=x^{(p)}(\varphi, \psi), y^{(p)}=$ $y^{(p)}(\varphi, \psi)$ and $\left(\sigma_{\alpha \beta}(x, y, \ldots)\right)_{\alpha, \beta=1,2}(p=\overline{1, \tilde{p}})[8,9]$ :

$$
\begin{aligned}
& \left\{\begin{array}{c}
\frac{\partial}{\partial \psi}\left(\frac{\sigma_{11} \sigma_{22}-\sigma_{21} \sigma_{12}}{\sigma_{11}} \frac{\partial x^{(p)}}{\partial \psi}-\frac{\sigma_{21}}{\sigma_{11}} \frac{\partial x^{(p)}}{\partial \varphi}\right)+\frac{\partial}{\partial \varphi}\left(\frac{1}{\sigma_{11}} \frac{\partial x^{(p)}}{\partial \varphi}+\frac{\sigma_{12}}{\sigma_{11}} \frac{\partial x^{(p)}}{\partial \psi}\right)=0 \\
\frac{\partial}{\partial \psi}\left(\frac{\sigma_{11} \sigma_{22}-\sigma_{21} \sigma_{12}}{\sigma_{22}} \frac{\partial y^{(p)}}{\partial \psi}+\frac{\sigma_{12}}{\sigma_{22}} \frac{\partial y^{(p)}}{\partial \varphi}\right)+\frac{\partial}{\partial \varphi}\left(\frac{1}{\sigma_{22}} \frac{\partial y^{(p)}}{\partial \varphi}-\frac{\sigma_{21}}{\sigma_{22}} \frac{\partial y^{(p)}}{\partial \psi}\right)=0
\end{array}\right. \\
& x^{(p)}\left(\varphi_{*}^{(p)}, \psi\right)=\tilde{x}\left(\tau_{*}^{(p)}(\psi)\right), \quad y^{(p)}\left(\varphi_{*}^{(p)}, \psi\right)=\tilde{y}\left(\tau_{*}^{(p)}(\psi)\right), \quad x^{(p)}\left(\varphi, Q^{(p)}\right)=\tilde{x}\left(\bar{\tau}^{(p)}(\varphi)\right), \\
& y^{(p)}\left(\varphi, Q^{(p)}\right)=\tilde{y}\left(\bar{\tau}^{(p)}(\varphi)\right), \quad x^{(p)}\left(\varphi^{*(p)}, \psi\right)=\tilde{x}\left(\tau^{*(p)}(\psi)\right), \quad y^{(p)}\left(\varphi^{*(p)}, \psi\right)=\tilde{y}\left(\tau^{*(p)}(\psi)\right), \\
& x^{(p)}(\varphi, 0)=\tilde{x}\left(\underline{\tau}^{(p)}(\varphi)\right), \quad y^{(p)}(\varphi, 0)=\tilde{y}\left(\underline{\tau}^{(p)}(\varphi)\right) ; \\
& y_{\varphi}^{\prime(p)}\left(\varphi_{*}^{(p)}, \psi_{*}^{(p)}\left(\tau_{1}, \ldots\right)\right)\left(\sigma_{11} y_{\psi}^{\prime(p)}\left(\varphi_{*}^{(p)}, \psi_{*}^{(p)}\left(\tau_{1}, \ldots\right)\right)-\sigma_{12} x_{\psi}^{\prime(p)}\left(\varphi_{*}^{(p)}, \psi_{*}^{(p)}\left(\tau_{1}, \ldots\right)\right)\right) \\
& -x_{\varphi}^{\prime(p)}\left(\varphi_{*}^{(p)}, \psi_{*}^{(p)}\left(\tau_{1}, \ldots\right)\right)\left(\sigma_{21} y_{\psi}^{\prime(p)}\left(\varphi_{*}^{(p)}, \psi_{*}^{(p)}\left(\tau_{1}, \ldots\right)\right)-\sigma_{22} x_{\psi}^{\prime(p)}\left(\varphi_{*}^{(p)}, \psi_{*}^{(p)}\left(\tau_{1}, \ldots\right)\right)\right)=0 \\
& y_{\varphi}^{\prime(p)}\left(\varphi^{*(p)}, \psi^{*(p)}\left(\tau_{2}, \ldots\right)\right)\left(\sigma_{11} y_{\psi}^{\prime(p)}\left(\varphi^{*(p)}, \psi^{*(p)}\left(\tau_{2}, \ldots\right)\right)-\sigma_{12} x_{\psi}^{\prime(p)}\left(\varphi^{*(p)}, \psi^{*(p)}\left(\tau_{2}, \ldots\right)\right)\right) \\
& -x_{\varphi}^{\prime(p)}\left(\varphi^{*(p)}, \psi^{*(p)}\left(\tau_{2}, \ldots\right)\right)\left(\sigma_{21} y_{\psi}^{\prime(p)}\left(\varphi^{*(p)}, \psi^{*(p)}\left(\tau_{2}, \ldots\right)\right)-\sigma_{22} x_{\psi}^{\prime(p)}\left(\varphi^{*(p)}, \psi^{*(p)}\left(\tau_{2}, \ldots\right)\right)\right)=0 \\
& y_{\varphi}^{\prime(p)}\left(\underline{\varphi}^{(p)}\left(\tau_{3}, \ldots\right), 0\right)\left(\sigma_{11} y_{\psi}^{\prime(p)}\left(\underline{\varphi}^{(p)}\left(\tau_{3}, \ldots\right), 0\right)-\sigma_{12} x_{\psi}^{\prime(p)}\left(\underline{\varphi}^{(p)}\left(\tau_{3}, \ldots\right), 0\right)\right) \\
& -x_{\varphi}^{\prime(p)}\left(\underline{\varphi}^{(p)}\left(\tau_{3}, \ldots\right), 0\right)\left(\sigma_{21} y_{\psi}^{\prime(p)}\left(\underline{\varphi}^{(p)}\left(\tau_{3}, \ldots\right), 0\right)-\sigma_{22} x_{\psi}^{\prime(p)}\left(\underline{\varphi}^{(p)}\left(\tau_{3}, \ldots\right), 0\right)\right)=0, \\
& y_{\varphi}^{\prime(p)}\left(\bar{\varphi}^{(p)}\left(\tau_{4}, \ldots\right), Q^{(p)}\right)\left(\sigma_{11} y_{\psi}^{\prime(p)}\left(\bar{\varphi}^{(p)}\left(\tau_{4}, \ldots\right), Q^{(p)}\right)-\sigma_{12} x_{\psi}^{\prime(p)}\left(\bar{\varphi}^{(p)}\left(\tau_{4}, \ldots\right), Q^{(p)}\right)\right) \\
& -x_{\varphi}^{\prime(p)}\left(\bar{\varphi}^{(p)}\left(\tau_{4}, \ldots\right), Q^{(p)}\right)\left(\sigma_{21} y_{\psi}^{\prime(p)}\left(\bar{\varphi}^{(p)}\left(\tau_{4}, \ldots\right), Q^{(p)}\right)-\sigma_{22} x_{\psi}^{\prime(p)}\left(\bar{\varphi}^{(p)}\left(\tau_{4}, \ldots\right), Q^{(p)}\right)\right)=0
\end{aligned}
$$

subject to conditions (3) and (4), where $\tau_{1} \in\left[\tau_{B}^{(p)} ; \tau_{A}^{(p)}\right], \tau_{2} \in\left[\tau_{D}^{(p)} ; \tau_{C}^{(p)}\right], \tau_{3} \in\left[\tau_{A}^{(p)} ; \tau_{D}^{(p)}\right], \tau_{4} \in$ $\left[\tau_{C}^{(p)} ; \tau_{B}^{(p)}\right] ; \tau=\tau_{*}^{(p)}(\psi), \tau=\bar{\tau}^{(p)}(\varphi), \tau=\tau^{*(p)}(\psi), \tau=\underline{\tau}^{(p)}(\varphi)\left(\varphi_{*}^{(p)} \leqslant \varphi \leqslant \varphi^{*(p)}, 0 \leqslant \psi \leqslant Q^{(p)}\right)$ are functions constructed by interpolating the experimental obtained their values $\tau_{* j_{*}}^{(p)}, \bar{\tau}_{\bar{i}}^{(p)}, \tau_{j^{*}}^{*(p)}, \underline{\tau}_{i}^{(p)}$ having some given $\psi_{* j_{*}}^{(p)}, \bar{\varphi}_{\bar{i}}^{(p)}, \psi_{j^{*}}^{*(p)}, \underline{\varphi}_{\underline{i}}^{(p)}$ at the sections $A_{p} B_{p}, B_{p} C_{p}, C_{p} D_{p}$ and $A_{p} D_{p}$, respectively.

We reconstruct the $\mathrm{CT}$, like in $[2,8,9]$, under the condition of minimizing the functional of the sum of the squares of the residuals of the expressions obtained from the generalized Cauchy-Riemann 
conditions, using the regularization concept

$$
\begin{gathered}
\Phi\left(x^{(1)}, y^{(1)}, \ldots, x^{(\tilde{p})}, y^{(\tilde{p})}, a_{s_{a}, 0}, b_{s_{b}, 0}, \ldots, a_{0, s_{a}}, b_{0, s_{b}}\right) \stackrel{d f}{=} \sum_{p=1}^{\tilde{p}}\left(\frac{\eta}{4}\left(\sum_{k_{a}, r_{a}=0}^{s_{a}, k_{a}} \frac{a_{k_{a}-r_{a}, r_{a}}^{2}}{100^{k_{a}}}+\sum_{k_{b}, r_{b}=0}^{s_{b}, k_{b}} \frac{b_{k_{b}-r_{b}, r_{b}}^{2}}{100^{k_{b}}}\right)\right. \\
+\left(\lambda_{1}\left(y_{\psi}^{\prime(p)} \cos \theta-x_{\psi}^{\prime(p)} \sin \theta\right)-y_{\varphi}^{\prime(p)} \sin \theta-x_{\varphi}^{\prime(p)} \cos \theta\right)^{2} \\
\left.+\left(\lambda_{2}\left(y_{\psi}^{\prime(p)} \sin \theta-x_{\psi}^{\prime(p)} \cos \theta\right)+y_{\varphi}^{\prime(p)} \cos \theta-x_{\varphi}^{\prime(p)} \sin \theta\right)^{2}\right) \rightarrow \min
\end{gathered}
$$

when

$$
0<\lambda_{2} \leqslant \lambda_{1}
$$

where $\eta$ is a regularization parameter [13], $w=100$ is a scaling coefficient.

The difference analogues of problem $(3),(4),(7)-(9)$ in the mesh domains $G_{z}^{\gamma(p)}(p=\overline{1, \tilde{p}})$, similarly to $[8-10]$, can be written in the following form:

$$
\begin{aligned}
& \left\{\begin{array}{c}
\left.x_{i+1, j}^{(p)}+x_{i-1, j}^{(p)}-2\left(1+\gamma^{(p) 2} B_{i, j}^{(p)}\right) x_{i, j}^{(p)}-x_{i-1, j+1}^{(p)}\right)+0.25 \gamma^{(p)} A_{i, j}^{(p)}\left(x_{i+1, j+1}^{(p)}+x_{i-1, j-1}^{(p)}-x_{i+1, j-1}^{(p)}\right) \\
\quad+C_{i, j}^{(p)}\left(x_{i+1, j}^{(p)}-x_{i-1, j}^{(p)}\right)+\gamma^{(p) 2} B_{i, j}^{(p)}\left(x_{i, j-1}^{(p)}+x_{i, j+1}^{(p)}\right)+\gamma^{(p)} D_{i, j}^{(p)}\left(x_{i, j+1}^{(p)}-x_{i, j-1}^{(p)}\right)=0, \\
y_{i+1, j}^{(p)}+y_{i-1, j}^{(p)}-2\left(1+\gamma^{(p) 2} B_{i, j}^{(p)}\right) y_{i, j}^{(p)}+0.25 \gamma^{(p)} A_{i, j}^{(p)}\left(y_{i+1, j+1}^{(p)}+y_{i-1, j-1}^{(p)}-y_{i+1, j-1}^{(p)}-y_{i-1, j+1}^{(p)}\right) \\
\quad+E_{i, j}^{(p)}\left(y_{i+1, j}^{(p)}-y_{i-1, j}^{(p)}\right)+\gamma^{(p) 2} B_{i, j}^{(p)}\left(y_{i, j-1}^{(p)}+y_{i, j+1}^{(p)}\right) \\
\quad+\gamma^{(p)} F_{i, j}^{(p)}\left(y_{i, j+1}^{(p)}-y_{i, j-1}^{(p)}\right)=0 \quad\left(1 \leqslant i \leqslant m^{(p)}, 1 \leqslant j \leqslant n^{(p)}\right) ;
\end{array}\right. \\
& x_{0, j}^{(p)}=\tilde{x}\left(\tau_{*}^{(p)}\left(\psi_{j}\right)\right), \quad y_{0, j}^{(p)}=\tilde{y}\left(\tau_{*}^{(p)}\left(\psi_{j}\right)\right), \quad x_{i, n}^{(p)}{ }^{(p)+1}=\tilde{x}\left(\bar{\tau}^{(p)}\left(\varphi_{i}\right)\right), \quad y_{i, n}^{(p)}+1=\tilde{y}\left(\bar{\tau}^{(p)}\left(\varphi_{i}\right)\right), \\
& x_{m^{(p)}+1, j}^{(p)}=\tilde{x}\left(\tau^{*(p)}\left(\psi_{j}\right)\right), \quad y_{m^{(p)}+1, j}^{(p)}=\tilde{y}\left(\tau^{*(p)}\left(\psi_{j}\right)\right), \quad x_{i, 0}^{(p)}=\tilde{x}\left(\underline{\tau}^{(p)}\left(\varphi_{i}\right)\right), \quad y_{i, 0}^{(p)}=\tilde{y}\left(\underline{\tau}^{(p)}\left(\varphi_{i}\right)\right) \\
& \left(0 \leqslant i \leqslant m^{(p)}+1, \quad 0 \leqslant j \leqslant n^{(p)}+1\right) ; \\
& \left(3 y_{0, j}^{(p)}-4 y_{1, j}^{(p)}+y_{2, j}^{(p)}\right)\left(\sigma_{11}\left(y_{0, j+1}^{(p)}-y_{0, j-1}^{(p)}\right)-\sigma_{12}\left(x_{0, j+1}^{(p)}-x_{0, j-1}^{(p)}\right)\right) \\
& -\left(\sigma_{21}\left(y_{0, j+1}^{(p)}-y_{0, j-1}^{(p)}\right)-\sigma_{22}\left(x_{0, j+1}^{(p)}-x_{0, j-1}^{(p)}\right)\right)\left(3 x_{0, j}^{(p)}-4 x_{1, j}^{(p)}+x_{2, j}^{(p)}\right)=0, \\
& \left(3 x_{m^{(p)}+1, j}^{(p)}-4 x_{m^{(p)}, j}^{(p)}+x_{m^{(p)}-1, j}^{(p)}\right)\left(\sigma_{21}\left(y_{m^{(p)}+1, j+1}^{(p)}-y_{m(p)+1, j-1}^{(p)}\right)-\sigma_{22}\left(x_{m^{(p)}+1, j+1}^{(p)}-x_{m^{(p)}+1, j-1}^{(p)}\right)\right) \\
& =\left(\sigma_{11}\left(y_{m^{(p)}+1, j+1}^{(p)}-y_{m^{(p)}+1, j-1}^{(p)}\right)-\sigma_{12}\left(x_{m^{(p)}+1, j+1}^{(p)}-x_{m^{(p)}+1, j-1}^{(p)}\right)\right)\left(3 y_{m^{(p)}+1, j}^{(p)}-4 y_{m^{(p)}, j}^{(p)}+y_{m^{(p)}-1, j}^{(p)}\right) \text {, } \\
& \left(y_{i+1,0}^{(p)}-y_{i-1,0}^{(p)}\right)\left(\sigma_{11}\left(3 y_{i, 0}^{(p)}-4 y_{i, 1}^{(p)}+y_{i, 2}^{(p)}\right)-\sigma_{12}\left(3 x_{i, 0}^{(p)}-4 x_{i, 1}^{(p)}+x_{i, 2}^{(p)}\right)\right) \\
& -\left(\sigma_{21}\left(3 y_{i, 0}^{(p)}-4 y_{i, 1}^{(p)}+y_{i, 2}^{(p)}\right)-\sigma_{22}\left(3 x_{i, 0}^{(p)}-4 x_{i, 1}^{(p)}+x_{i, 2}^{(p)}\right)\right)\left(x_{i+1,0}^{(p)}-x_{i-1,0}^{(p)}\right)=0, \\
& \left(y_{i+1, n^{(p)}+1}^{(p)}-y_{i-1, n^{(p)}+1}^{(p)}\right)\left(\sigma_{11}\left(3 y_{i, n^{(p)}+1}^{(p)}-4 y_{i, n^{(p)}}^{(p)}+y_{i, n^{(p)}-1}^{(p)}\right)\right. \\
& \left.-\sigma_{12}\left(3 x_{i, n^{(p)}+1}^{(p)}-4 x_{i, n^{(p)}}^{(p)}+x_{i, n^{(p)}-1}^{(p)}\right)\right)-\left(\sigma_{21}\left(y_{i, n^{(p)}-1}^{(p)}-4 y_{i, n^{(p)}}^{(p)}+3 y_{i, n^{(p)}+1}^{(p)}\right)\right. \\
& \left.-\sigma_{22}\left(3 x_{i, n^{(p)}+1}^{(p)}-4 x_{i, n^{(p)}}^{(p)}+x_{i, n^{(p)}-1}^{(p)}\right)\right)\left(x_{i+1, n^{(p)}+1}^{(p)}-x_{i-1, n^{(p)}+1}^{(p)}\right)=0, \quad i=\overline{0, m^{(p)}+1}, j=\overline{0, n^{(p)}+1},
\end{aligned}
$$

where $\gamma^{(p)}$ are quasiconformal invariants [10] for corresponding domains $G_{\omega}^{\gamma(p)}=\left\{\left(\varphi_{i}^{(p)}, \psi_{j}^{(p)}\right): \varphi_{i}^{(p)}=\right.$ $\varphi_{*}^{(p)}+i \Delta \varphi^{(p)}, i=\overline{0, m^{(p)}+1} ; \psi_{j}^{(p)}=j \Delta \psi^{(p)}, j=\overline{0, n^{(p)}+1} ; \Delta \psi^{(p)}=Q^{(p)} /\left(n^{(p)}+1\right), \Delta \varphi^{(p)}=\left(\varphi^{*(p)}-\right.$ $\left.\left.\varphi_{*}^{(p)}\right) /\left(m^{(p)}+1\right), \gamma^{(p)}=\Delta \varphi^{(p)} / \Delta \psi^{(p)}, m^{(p)}, n^{(p)} \in N\right\} ; x_{i, j}^{(p)}=x^{(p)}\left(\varphi_{i}^{(p)}, \psi_{j}^{(p)}\right), y_{i, j}^{(p)}=y^{(p)}\left(\varphi_{i}^{(p)}, \psi_{j}^{(p)}\right)$, 
$z_{i, j}^{(p)}=z^{(p)}\left(\varphi_{i}^{(p)}, \psi_{j}^{(p)}\right), \sigma_{\alpha \beta_{i, j}}^{\gamma(p)}=\sigma_{\alpha \beta}\left(x_{i, j}^{(p)}, y_{i, j}^{(p)}\right), \lambda_{i, j}^{\gamma(p)}=\lambda\left(x_{i, j}^{(p)}, y_{i, j}^{(p)}\right) ; A_{i, j}^{(p)}, B_{i, j}^{(p)}, C_{i, j}^{(p)}, D_{i, j}^{(p)}, E_{i, j}^{(p)}, F_{i, j}^{(p)}$ are determined in the same way as in [10].

We rewrite the functional (10) and conditions (11) for all internal nodal points $\left(x_{i, j}^{(p)}, y_{i, j}^{(p)}\right)(p=\overline{1, \tilde{p}}$, $\left.i=\overline{1, m^{(p)}}, j=\overline{1, n^{(p)}}\right)$ as follows:

$$
\begin{gathered}
\Phi\left(x_{0,0}^{(1)}, y_{0,0}^{(1)}, \ldots, x_{m^{(\tilde{p})}+1, n^{(\tilde{p})}+1}^{(\tilde{p})}, y_{m^{(\tilde{p})}+1, n(\tilde{p})+1}^{(\tilde{p})}, a_{s_{a}, 0}, \ldots, a_{0, s_{a}}, b_{s_{b}, 0}, \ldots, b_{0, s_{b}}\right) \\
=\sum_{p, i, j=1}^{\tilde{p}, m^{(p)}, n^{(p)}}\left(\eta \Delta \varphi^{(p) 2}\left(\sum_{k_{a}, r_{a}=0}^{s_{a}, k_{a}} \frac{a_{k_{a}-r_{a}, r_{a}}^{2}}{w^{k_{a}}}+\sum_{k_{b}, r_{b}=0}^{s_{b}, k_{b}} \frac{b_{k_{b}-r_{b}, r_{b}}^{2}}{w^{k_{b}}}\right)\right. \\
+\left(\lambda_{1_{i, j}} \gamma^{(p)}\left(\left(y_{i, j+1}^{(p)}-y_{i, j-1}^{(p)}\right) \cos \theta_{i, j}-\left(x_{i, j+1}^{(p)}-x_{i, j-1}^{(p)}\right) \sin \theta_{i, j}\right)-\left(y_{i+1, j}^{(p)}\right.\right. \\
\left.\left.-y_{i-1, j}^{(p)}\right) \sin \theta_{i, j}-\cos \theta_{i, j}\left(x_{i+1, j}^{(p)}-x_{i-1, j}^{(p)}\right)\right)^{2}+\left(\left(x_{i, j-1}^{(p)}-x_{i, j+1}^{(p)}\right) \cos \theta_{i, j}+\left(y_{i, j+1}^{(p)}-\right.\right. \\
\left.\left.\left.\left.-y_{i, j-1}^{(p)}\right) \sin \theta_{i, j}\right) \lambda_{2_{i, j}} \gamma^{(p)}+\left(y_{i+1, j}^{(p)}-y_{i-1, j}^{(p)}\right) \cos \theta_{i, j}-\left(x_{i+1, j}^{(p)}-x_{i-1, j}^{(p)}\right) \sin \theta_{i, j}\right)^{2}\right) \rightarrow \min \\
0<\lambda_{2_{i, j}}^{\gamma(p)} \leqslant \lambda_{1_{i, j}}^{\gamma(p)}
\end{gathered}
$$

where $a_{k_{a}^{\prime}-r_{a}^{\prime}, r_{a}^{\prime}}, b_{k_{b}^{\prime}-r_{b}^{\prime}, r_{b}^{\prime}}\left(k_{a}^{\prime}=\overline{0, s_{a}}, r_{a}^{\prime}=\overline{0, k_{a}^{\prime}}, k_{b}^{\prime}=\overline{0, s_{b}}, r_{b}^{\prime}=\overline{0, k_{b}^{\prime}}\right)$ are parameters for which values are sought. It seems reasonable to solve the problem of nonlinear programming (15), (16) by one of the appropriate methods of local optimization, e.g., by penalty function method, implemented in the [14] with the application of parallelization mechanisms.

In addition, given the necessity for a huge amount of calculations when refining the coordinates of the mesh nodes, it is advisable to parallelize the corresponding computations. One of the promising mechanisms that provide such capabilities is the CUDA platform [15]. In particular, it is advisable to parallelize the procedure of solving systems of equations (12) with the use of "red-black" ordering of the unknowns [16]. Such order of "perambulation" ensures that both nodal coordinates from the previous steps and the approximate values are used in the iterative process, according to the Seidel method.

The algorithm for solving the original problem is similar to [9]. The main difference lies in applying the optimization problem in the form of (15), (16). We also propose to refine the parameters of the meshes by the formula (12) in parallel, namely: firstly at the nodes with indices for which the condition $(i+j) / 2 \in \mathbb{N}$ is fulfilled, and then in all other cases $\left(p=\overline{1, \tilde{p}}, i=\overline{1, m^{(p)}}, j=\overline{1, n^{(p)}}\right)$. Also, all of the notes given in [9] remain valid.

\section{Numerical results}

We present the results of numerical experiments of imitative restoration of media structure using the following input data: $\tilde{x}(\tau)=150 \cos \tau, \tilde{y}(\tau)=100 \sin \tau, \tilde{p}=20, m^{(p)}=100, s_{a}=s_{b}=3$, $a_{0,0}=b_{0,0}=1, a_{k_{a}-r_{a}, r_{a}}=0, b_{k_{b}-r_{b}, r_{b}}=0\left(k_{a}=\overline{1, s_{a}}, r_{a}=\overline{0, k_{a}}, k_{b}=\overline{1, s_{b}}, r_{b}=\overline{0, k_{b}}\right), \eta=100$, $q=200, \varepsilon_{1}=\varepsilon_{2}=10^{-2}, \varphi_{*}^{(p)}=0, \varphi^{*(p)}=1, \tau_{A}^{(p)}=((p-1) / \tilde{p}+9 / 8) \pi, \tau_{B}^{(p)}=\tau_{A}^{(p)}-\pi / 4$, $\tau_{C}^{(p)}=\tau_{A}^{(p)}-\pi, \tau_{D}^{(p)}=\tau_{C}^{(p)}-\pi / 4, p=\overline{1, \tilde{p}}$ (here the parameters $\tau_{A}^{(p)}, \tau_{B}^{(p)}, \tau_{C}^{(p)}, \tau_{D}^{(p)}$ are chosen similarly to the polar scheme of quasipotential application [1]),

$\theta=0.7+8 \cdot 10^{-3} x-4 \cdot 10^{-3} y-4 \cdot 10^{-5} x^{2}+10^{-4} x y-7 \cdot 10^{-5} y^{2}-3 \cdot 10^{-7} x^{3}-8 \cdot 10^{-7} x^{2} y-17 \cdot 10^{-7} x y^{2}+14 \cdot 10^{-7} y^{3}$.

Parameter values $Q^{(p)}, \Psi_{* j}^{(p)}, \Psi_{j}^{*(p)}, \bar{\varphi}_{i}^{(p)}, \underline{\varphi}_{i}^{(p)}(1 \leqslant p \leqslant \tilde{p})$ are obtained by the following algorithm: solve $\tilde{p}$ problems of modeling the quasiideal stream (4)-(6), (7) using the method described, e.g., in [10] (with simultaneous $Q^{(p)}$ determination) having given

$\bar{\lambda}_{1}=\bar{\lambda}_{1}(x, y)=1.5-8 \cdot 10^{-4} x-5 \cdot 10^{-4} y+10^{-5} x y+3 \cdot 10^{-5} y^{2}+10^{-7} x^{3}+10^{-7} x^{2} y+2 \cdot 10^{-7} x y^{2}-2 \cdot 10^{-7} y^{3}$,

Mathematical Modeling and Computing, Vol. 6, No. 2, pp. 211-219 (2019) 
$\bar{\lambda}_{2}=\bar{\lambda}_{2}(x, y)=1+2 \cdot 10^{-3} x-10^{-3} y-10^{-5} x^{2}+4 \cdot 10^{-5} x y+3 \cdot 10^{-5} y^{2}+10^{-8} x^{3}-10^{-8} x^{2} y+10^{-8} x y^{2}+10^{-7} y^{3} ;$

carry out a uniform division of the domain boundary into $s(s=60)$ points, in each of which determine the values of the corresponding function $\psi_{* j}^{(p)}, \psi_{j}^{*(p)}, \bar{\varphi}_{i}^{(p)}, \underline{\varphi}_{i}^{(p)}$; calculate $\Psi_{* j}^{(p)}, \Psi_{j}^{*(p)}$ according to the difference representations of formulas $\Psi_{*}^{(p)}(M)=\partial \psi_{*}^{(p)}(M) / \partial l, \Psi^{*(p)}(M)=\partial \psi^{*(p)}(M) / \partial l$. The problem (1)-(4) is solved using the above data and implementation of the proposed algorithm where, in particular, the parameters of expressions (4) are identified:

$$
\begin{aligned}
\lambda_{1} \approx & 1.35+2.8 \cdot 10^{-3} x+4.7 \cdot 10^{-3} y-9 \cdot 10^{-6} x^{2}+3.3 \cdot 10^{-5} x y+7.2 \cdot 10^{-5} y^{2}-5 \cdot 10^{-8} x^{3} \\
& -5 \cdot 10^{-7} x^{2} y-3.6 \cdot 10^{-7} x y^{2}-6.2 \cdot 10^{-7} y^{3} \\
\lambda_{2} \approx & 1.15+2.2 \cdot 10^{-3} x+2.7 \cdot 10^{-3} y-2.1 \cdot 10^{-5} x^{2}+4 \cdot 10^{-5} x y+5 \cdot 10^{-6} y^{2}-7 \cdot 10^{-8} x^{3} \\
& -1.4 \cdot 10^{-7} x y^{2}-5.2 \cdot 10^{-7} y^{3} .
\end{aligned}
$$

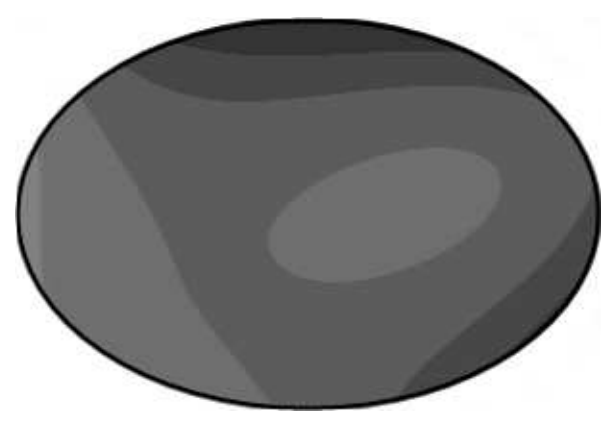

$a$

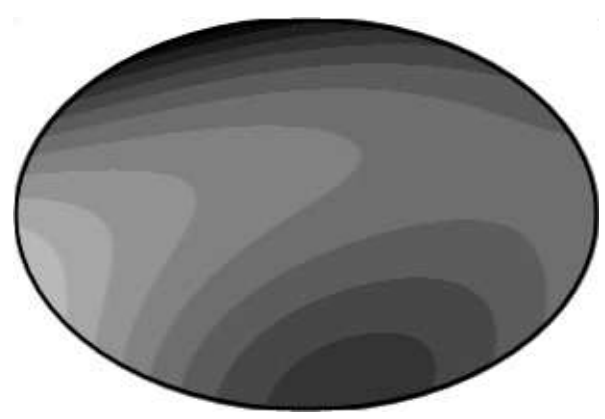

C

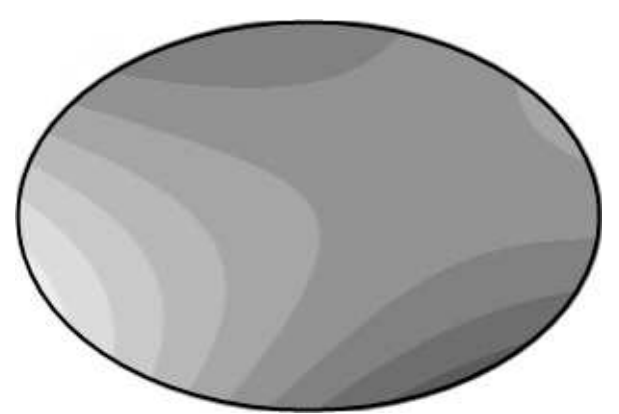

$b$

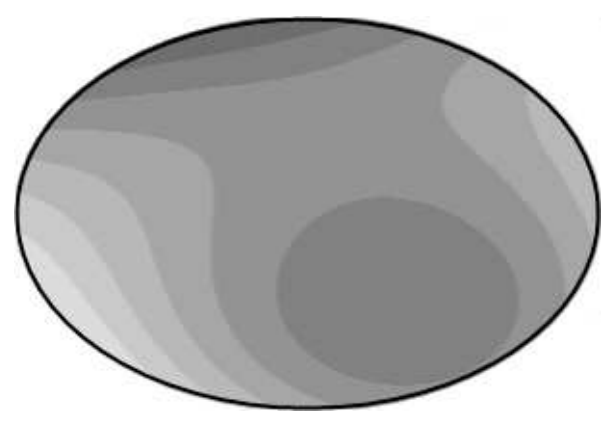

$d$

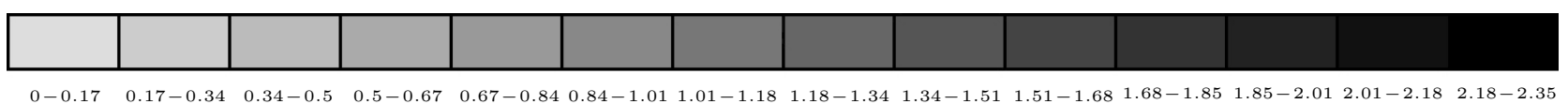

Fig. 2. Distributions of $\lambda_{1}$ and $\lambda_{2}$ : exact $(\boldsymbol{a})$ and $(\boldsymbol{b})$, and approximated $(\boldsymbol{c})$ and $(\boldsymbol{d})$ solutions, respectively.

The values of calculated $\lambda_{1}$ and $\lambda_{2}$, and exact (etalon) $\bar{\lambda}_{1}$ and $\bar{\lambda}_{2}$ correspond to the grayscale in Fig. $2 c$ and Fig. $2 d$, and Fig. $2 a$ and Fig. $2 b$, respectively. We see that residual peaks are reached at eight specific points $\Delta \lambda_{1}(-138.924,37.713)=-0.611, \Delta \lambda_{1}(-90.252,-79.874)=0.513, \Delta \lambda_{1}(2.15,99.99)=$ $0.369, \Delta \lambda_{1}(141.314,33.536)=-0.413, \Delta \lambda_{2}(-147.411,18.5)=0.134, \Delta \lambda_{2}(-8.107,61)=0.337$, $\Delta \lambda_{2}(-20.104,-99.098)=0.668, \Delta \lambda_{2}(149.519,8.005)=-0.338$, most of which are located at the domain boundary, where, apparently, quasiconformal errors occur. Certainly, the first of these peaks is caused by the insufficient value of the reconstructed CT $\lambda_{1}$, and all others are characterized by displaced inhomogeneity locations. The average residual modulus in the domain is $\Delta \tilde{\lambda}_{1}=0.179$, $\Delta \tilde{\lambda}_{2}=0.17$. 


\section{Conclusions}

Given the relevance of developing the complex analysis methods for solving AQT problems, the corresponding algorithm is modified to the case of parameter identification of anisotropic media having more specific a priori information about eigendirections of the sought CT. In this, the image reconstruction is carried out using additional data regarding the longer axis of the corresponding anisotropy ellipse. The application of this type of data is especially common in medicine, geology, etc. Certainly, like in [9], the basis of the described approach is taking into account the distribution of both local velocity values along the sections of input and output particles from the investigated object, and potentials at remaining places of domain border on the one hand, and applying the quasiconformal mapping methods to ensure the possibility of solving the direct problems in the iterative process on the other. However, in comparison to [9], parallelization of calculations is introduced here, and in order to reduce the number of intermediate calculations, the form of minimizing functional is optimized. Here, when imposing additional restrictions on the eigendirections of the $\mathrm{CT}$, the adaptability of the corresponding algorithm to a series of specific cases of practice is ensured.

The developed algorithm is characterized by comparatively fast computer convergence (since, unlike many methods used, it does not require finding numerical derivatives of the CT distribution function at certain points and refining the boundary nodes at each iteration step) and relative ease of its parallelization. The latter is implemented with the use of "red-black" ordering of the unknowns, mechanisms of the CUDA platform, and the ALGLIB library. It should be noted that the anisotropy tensor affects the deterioration order of accuracy and stability of the corresponding algorithm, which, in particular, requires both the creation of special constructions-procedures of Tikhonov-type regularization and approaches to optimization (in particular, parallelization) of calculations.

We plan to extend our algorithm for the following cases: possibility of spatial reconstruction of the CT, parameter identification of piecewise-homogeneous and piecewise-inhomogeneous media, in particular, using the conditions of non-ideal contact [17] both inside the object under study and at the applied quasipotential sections (the latter allows to implement the complete electrode model given in $[1,4,5]$ ), taking into account the distribution of impedance in the body (see, e.g. [1]), and also the use of three-component (device for collecting and entering physical data, calculating devices, and server) GRID-technologies [18].

[1] Holder D. Electrical Impedance Tomography. Methods, History and Applications. London, Institute of Physics (2005).

[2] Herwanger J. V., Pain C. C., Binley A., De Oliveira C. R.E., Worthington M. H. Anisotropic resistivity tomography. Geophysical Journal International. 158 (2), 409-425 (2004).

[3] Abascal J.-F. P. J., Lionheart W. R. B., Arridge S. R., Schweiger M., Atkinson D., Holder D. S. Electrical impedance tomography in anisotropic media with known eigenvectors. Inverse Problems. 27 (6), 1-17 (2011).

[4] Crabb M. EIT Reconstruction Algorithms for Respiratory Intensive Care. PhD thesis. Manchester, University of Manchester (2014).

[5] Tallman T. N. Conductivity-Based Nanocomposite Structural Health Monitoring via Electrical Impedance Tomography. PhD thesis. Ann Arbor, University of Michigan (2015).

[6] Calderón A. P. On an inverse boundary value problem. Computational and Applied Mathematics. 25 (2-3), 133-138 (2006).

[7] Martins T.C., Tsuzuki M.S. G. Investigating anisotropic EIT with simulated annealing. IFACPapersOnLine. 50 (1), 9961-9966 (2017).

[8] Bomba A., Boichura M. Numerical complex analysis method for parameters identification of anisotropic media using applied quasipotential tomographic data. Part 1: Problem statement and its approximation. Mathematical and Computer Modelling. Series: Physical and Mathematical Sciences. 18 (1), 14-24 (2018). 
[9] Bomba A., Safonyk A., Michuta O., Boichura M. Applied quasipotential method for solving the coefficient problems of parameter identification of anisotropic media. Informatics, Control, Measurement in Economy and Environment Protection. 9 (1), 33-36 (2019).

[10] Bomba A., Kashtan S. On one method for constructing a dynamical mesh of nonlinear quasiperfect processes in deformable anisotropic media. Journal of Applied Computer Science. 12 (2), 7-21 (2004).

[11] Astala K., Päivärinta L., Lassas M. Calderón's inverse problem for anisotropic conductivity in the plane. Communications in Partial Differential Equations. 30 (1-2), 207-224 (2005).

[12] Lionheart W. R. B. Conformal uniqueness results in anisotropic electrical impedance imaging. Inverse Problems. 13 (1), 125-134 (1997).

[13] Tikhonov A. N., Arsenin V. Y. Solution of Ill-posed Problems. New York, Wiley (1977).

[14] http://www.alglib.net/

[15] https://developer.nvidia.com/cuda-zone/

[16] Konstantinidis E., Cotronis Y. Graphics processing unit acceleration of the red/black SOR method. Concurrency and Computation: Practice and Experience. 25 (8), 1107-1120 (2013).

[17] Martyniuk P. M., Michuta O. R., Ulianchuk-Martyniuk O. V., Kuzlo M. T. Numerical investigation of pressure head jump values on a thin inclusion in one-dimensional non-linear soil mousture transport problem. International Journal of Applied Mathematics. 31 (4), 649-660 (2018).

[18] Murugananthi C., Ramyachitra D. Life science applications in grid environment. Proceedings on International Conference on Research Trends in Computer Technologies. Coimbatore, India. 1-5 (2013).

\title{
Метод реконструкції зображень анізотропних середовищ за даними томографії прикладених квазіпотенціалів
}

\author{
Бомба А. Я., Кузло М. Т., Мічута О. Р., Бойчура М. В. \\ Начіональний університет водного господарства та природокористування, \\ вул. Соборна, 11, Рівне, 33028, Україна
}

\begin{abstract}
Модифіковано алгоритм розв'язання коефіцієнтних задач ідентифікації параметрів анізотропних середовищ за даними томографії прикладних квазіпотенціалів на випадок наявності конкретнішої апріорної інформації щодо власних напрямків відповідного тензора провідності. Застосування доволі поширене на практиці, зокрема в медицині об'єктом таких досліджень можуть бути середовища із ділянками волокнистості чи шаруватості (до яких належать м'язи, кістки тощо), в яких існують потоки некулястих частинок (наприклад, еритроцитів). В основу відповідного алгоритму й надалі покладено почергове розв'язання задач на квазіконформні відображення та ідентифікацію параметрів, проте у цій роботі його доповнено процедурою розпаралелення обчислень і "пришвидшено" задачу оптимізації. Останне характеризується значним зменшенням кількості проміжних розрахунків та, у разі накладання додаткових обмежень на власні напрямки тензора провідності, приводить до можливості оптимальної пристосовності алгоритму до конкретних випадків практики. Наведено відповідні результати числових експериментів імітаційного відновлення структури середовища.
\end{abstract}

Ключові слова: томографія прикладених квазіпотениіалів, квазіконформні відображення, анізотропія, ідентифікачія, нелінійні обернені задачі.

2000 MSC: 30C62, 35J25, 65E05, 65N21

Удк: 519.6 